\title{
Exact number concepts are limited to the verbal count range
}

\author{
Benjamin Pitt \\ pitt@berkeley.edu
}

\author{
Edward Gibson \\ ebigson@mit.edu
}

\author{
Steven T. Piantadosi \\ stp@berkeley.edu
}

\begin{abstract}
Previous studies suggest that mentally representing exact numbers larger than four depends on a verbal count routine (e.g. "one, two, three..."). However, these findings are controversial, as they rely on comparisons across radically different languages and cultures. We tested the role of language in number concepts within a single population - the Tsimane' of Bolivia - where knowledge of number words varies across individual adults. We used a novel data analysis model to quantify the point at which participants $(\mathrm{N}=30)$ switched from exact to approximate number representations during a simple numerical matching task. The results show that these behavioral switchpoints were bounded by participants' verbal count ranges; their representations of exact cardinalities were limited to the number words they knew. Beyond that range, they resorted to numerical approximation. These findings resolve competing accounts of previous findings and provide unambiguous evidence that large exact number concepts are enabled by language.
\end{abstract}

\section{Statement of Relevance}

Animal species share some basic numerical abilities, but only humans can reason about exact numbers like seven or 42. What allows us to accomplish this extraordinary cognitive feat? Here we tested the role of language in number concepts among the Tsimane', an indigenous group whose adults vary in their knowledge of number words. We found that this linguistic knowledge placed an upper bound on participants' ability to mentally represent exact quantities; participants correctly matched the number of objects in (unlabeled) sets only when that number was within their highest verbal count. For larger sets, they had no way of representing exactly how many objects they saw. This finding provides the clearest evidence to date that number words play a functional role in people's ability to represent exact quantities larger than four, and supports the broader claim that language can enable new conceptual abilities. 


\section{Introduction}

Language gives humans extraordinary cognitive abilities, but its role in numerical cognition remains unresolved. Studies of human infants and non-human animals have shown that at least some numerical abilities do not depend on language. Babies, monkeys, and even invertebrates can make precise distinctions between small quantities without counting (up to about four; Feigenson, Dehaene, \& Spelke, 2004; Pahl, Si, \& Zhang, 2013) and can rapidly distinguish the numerosities of larger sets, although only roughly (Dehaene, 1997; Dehaene, Dehaene-Lambertz, \& Cohen, 1998; Cheyette \& Piantadosi, 2020). Whereas the ability to represent small exact and large approximate numbers is conserved across species, the ability to represent larger numbers exactly (e.g. exactly seven) appears to be unique to humans (Dehaene, 1997; c.f. Brannon, 2005) and is often attributed to language (Bloom, 1994; Chomsky, Keyser, et al., 1988; Carey \& Barner, 2019). Specifically, predominant accounts posit that the structure of the verbal count list (e.g. "one, two, three..."), which children learn to recite long before they understand the meanings of the number words (Wynn, 1992; Davidson, Eng, \& Barner, 2012; Sarnecka, Goldman, \& Slusser, 2015), allows them to discover the logic of number by induction (Carey, 2004, 2009; Spelke, 2003; Piantadosi, Tenenbaum, \& Goodman, 2012; Bloom, 1994; cf. Gelman \& Gallistel, 2004; Leslie, Gelman, \& Gallistel, 2008; Butterworth, Reeve, Reynolds, \& Lloyd, 2008).

This account draws support from studies of isolated groups with few or no words for exact quantities. Specifically, two indigenous groups in the Brazilian Amazon the Pirahã and the Mundurukú - have no words denoting large exact quantities (and in the case of the Pirahã, no words for any exact quantity, not even one; Frank, Everett, Fedorenko, \& Gibson, 2008; Pica, Lemer, Izard, \& Dehaene, 2004). To test large exact number concepts in such groups without using number words, researchers have used simple numerical tasks that require only behavioral responses, often on sets of physical objects (e.g. 7 pebbles; Figure 1). Pirahã and Mundurukú adults perform well on these tasks only up to about four; for larger cardinalities, they are unable to reproduce the number of objects in a set exactly, relying instead on approximation (Gordon, 2004; Frank et al., 2008; Pica et al., 2004). A similar pattern has been found in Nicaraguan Homesigners, a group of congenitally deaf adults whose language lacks a count routine (Spaepen, Coppola, Spelke, Carey, \& Goldin-Meadow, 2011). In sum, people without words for large exact numbers seem unable to represent cardinalities larger than four, leading some scholars to conclude that the verbal count list "enables exact enumeration" (Gordon, 2004).

However, these findings are difficult to interpret (e.g. Gleitman \& Papafragou, 2012; Butterworth et al., 2008), in part because they rely on comparing across languages and cultures. Groups without exact number words (like the Pirahã) are compared, if only implicitly, to groups with productive counting systems (like Americans). Of course, isolated groups differ radically from Western, Educated, Industrialized, Rich, and Democratic (WEIRD; Henrich, Heine, \& Norenzayan, 2010) groups in many ways besides in their knowledge of number words (e.g. Everett, 2009), and any of these differences could account for the observed difference in numerical cognition (Gelman \& Butterworth, 2005; Spaepen et al., 2011). For example, some scholars suggest that the Pirahã failed to make exact numerical matches of large sets because they were simply "indifferent to exact numerical equality" (Gelman \& Gallistel, 2004; also see Laurence \& Margolis, 2007; Spaepen et al., 2011), perhaps because "keeping track of large exact quantities is not critical for getting along in Pirahã society" (Casasanto, 2005). Indeed, whereas quantification is prized in WEIRD cultures, some unindustrialized groups like the Pirahã do not track chronological age, use currency, or have units of measurement (Cooperrider \& Gentner, 2019; Everett, 2009; Diekmann et al., 2017). Cross-cultural comparisons cannot in principle distinguish whether large exact number concepts depend on a verbal count routine or on other aspects of language and culture.

Even if these studies had clearly established a causal role for language in large exact number concepts, it would remain unclear what role that is. Some accounts posit that the verbal count list is instrumental both for inducing the principles of number (e.g. Hume's principle; Carey \& Barner, 2019; Schneider \& Barner, 2020) and for using those principles to construct representations of specific cardinalities (e.g. exactly seven; Carey, 2004). Alternatively, the verbal count list may be necessary for inducing the logic of number only, which people could then use to enumerate large sets whether or not the corresponding verbal symbols were available to them. Previous cross-cultural studies cannot distinguish between these possibilities because they test numerical abilities only at the extremes; the numerical abilities of the Pirahã (or any group without large exact number words) could reflect a lack of the requisite number principles, number words, or both.

Some studies have tested the role of number words in large exact number concepts without comparing across language groups, with mixed results. In a group of MIT undergraduates, verbal interference impaired performance on some numerical tasks more than a spatial control task, suggesting a functional role for language in representing large exact numbers (Frank, Fedorenko, Lai, Saxe, \& Gibson, 2012). However, despite verbal interference, participants performed well on two other tests of large exact number representations, including the orthogonal matching task, complicating interpretation of the results. In another study, US children overwhelmingly failed to make exact numeri- 
cal matches of large sets, but they were also imprecise in a task that only required one-to-one matching of objects (Schneider \& Barner, 2020). In sum, previous studies do not clearly establish whether or how large exact number concepts are shaped by language.

Here we addressed these inferential challenges by testing the relationship between number words and number concepts in the Tsimane', a group of unindustrialized farmer-foragers indigenous to the Bolivian Amazon (Huanca, 2008). Unlike other isolated populations, the Tsimane' have a fully productive system of number words in their language. Yet, unlike adults in WEIRD cultures, Tsimane' adults exhibit considerable variation in their knowledge of the verbal count list; many Tsimane' adults can count indefinitely, but some do not know words above 10, others falter at 12 , etc. This variability allowed us to (a) compare verbal and numerical abilities across individuals, rather than across groups and (b) to do so at many intermediate levels, not just at the extremes. To determine which large numbers participants could represent exactly, and which numbers they could only approximate, we used a novel statistical analysis to model participants' behavioral responses in a numerical matching task. This model uses the known psychophysical properties of numerical estimation to determine the set size at which participants switched from exact to approximate number representations. By comparing this switchpoint to participants' highest verbal counts, we tested whether people need a system of number symbols (like those in the verbal count list) in order to represent large exact numbers. If they do (Carey, 2009; Spelke, 2003), then we should find not only that these abilities are correlated, but that one systematically exceeds the other; participants' highest verbal counts should place an upper bound on their numerical representations. Alternatively, if number words are necessary for discovering the logic of number, but not for deploying it (or not at all, e.g. Leslie et al., 2008; Butterworth et al., 2008), then participants' numerical representations should sometimes exceed their verbal count ranges. Unlike in previous studies, here the relationship between verbal counting and numerical reproduction cannot be attributed to broad cultural or linguistic differences, since our participants shared the same culture, language, and in many cases lived in the same small community.

\section{Method}

\section{Participants}

All participants gave verbal informed consent before participating in the study, which was approved by the institu- tional review board at [redacted]. As part of an initial questionnaire, participants were asked to count aloud as high as they were able, starting at one, in whatever language they preferred (i.e. Tsimane' or Spanish). Participants who faltered in their count routine for numbers below 20 were selected for the low-counter group $(\mathrm{N}=15$; mean age $=$ $48.73+/-4.34$ years, mean schooling $=0.2+/-.11$ years) and those who counted past 40 were selected for the control group of high counters $(\mathrm{N}=15$; mean age $=32.87+/$ 4.52 years, mean schooling $=4.00+/-.65$ years). The sample size was determined by the number of participants we encountered during our limited time in the field who qualified as low-counters (with a maximum of 20). After this initial selection, we tested participants' count ranges using a pebble-counting task. After this initial selection, we tested participants' count ranges using a pebble-counting task, described below.

\section{Pebble-counting task}

Participants were presented with a pile of glass pebbles ( $\mathrm{N}=30$ for low counters, $\mathrm{N}=40$ for high counters) on the testing table. Starting with the pebbles on their left side, participants moved them one at a time to the right while counting each one aloud, in the language of their choosing. ${ }^{1}$ After they stopped counting, participants were asked how many pebbles there were in the counted set. The experimenter(s) and translator noted any counting errors. With three exceptions, participants performed this task twice, once before and once after completing the matching tasks. We used the higher of the two counts as participants' highest verbal count.

\section{Numerical matching tasks}

Participants then performed two non-verbal number tasks in which they were asked to make arrays with the same number of objects as a sample array. In the parallel matching task, the experimenter presented a sample array of objects (in a lateral line) for each trial and participants arranged their response array parallel to each sample array (see Figure 1, top left; see Supplementary Materials for details). Because sample and response arrays were parallel, participants could use 1-1 correspondence to perform the match in this task, spatially aligning each object in their response array with an object in the sample array without representing the cardinality of either set. For this reason, the parallel matching task does not test representations of large exact numbers. Rather, success on this task suggests understanding of exact numerical equivalence: for two sets to be equal in number, every element in each set must correspond to an element in the other set (Jara-Ettinger,

\footnotetext{
${ }^{1}$ Both Tsimane' and Spanish have recursive, base-10 counting systems. Unlike English and Spanish, which have irregular constructions like eleven (11) or trece (13), Tsimane' number words are regular throughout the teens (i.e. ten-one, ten-two, ten-three... ). Some participants used a mixture of both languages (e.g. starting in Tsimane' for the lower numbers and then switching into Spanish for higher numbers) whereas other participants used only one language to count.
} 
Piantadosi, Spelke, Levy, \& Gibson, 2017; Hume, 1978; Schneider \& Barner, 2020). This task also functioned as a comprehension check, ensuring that participants understood the mechanics of these numerical reproduction tasks. Participants correctly produced a parallel match for each of five sample arrays $(\mathrm{N}=3,4,5,10$, and 15$)$ and then advanced to the orthogonal matching task.

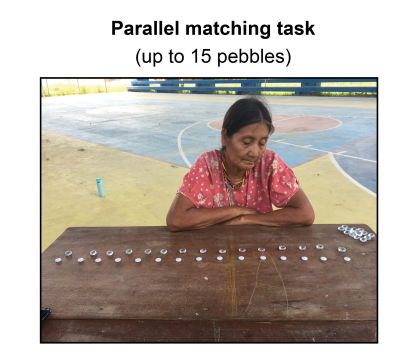

Parallel matching results Low-counters

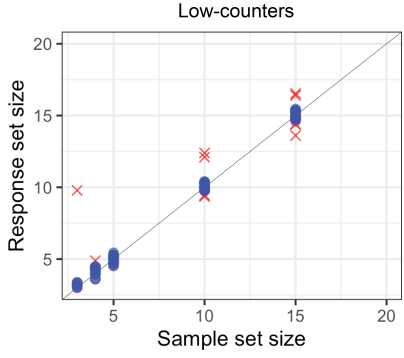

Correct $\times$ Incorrect

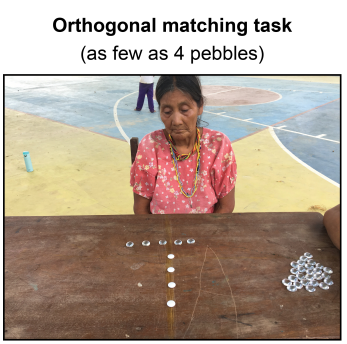

Orthogonal matching results Low-counters

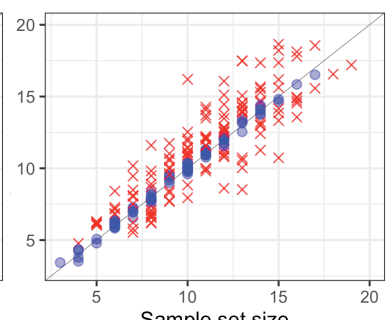

Sample set size
Figure 1: Numerical matching tasks. In the parallel matching task (top left), Tsimane' participants used 1-to-1 correspondence to make a numerical match (on sets of $3,4,5,10$, and 15 white pebbles). Low-counters were highly accurate on this task (bottom left). In the orthogonal matching task (top right), correctly matching required participants to represent the cardinality of the sets (of 4-25 white pebbles). Low-counters' accuracy was variable on this task, with signs of scalar variability (bottom right).

In the orthogonal matching task, the sample arrays were arranged sagittally in a line extending away from the participant. Participants arranged their response arrays laterally (as in the parallel matching task), in a line that was orthogonal to the sample array (Figure 1, top right; see Supplementary Materials for details). Unlike the parallel matching task, this task precludes spatially aligning sample and response arrays, and therefore requires participants to represent the cardinality of each set. However, it is minimally demanding on participants' numerical abilities: Participants were not asked to perform any arithmetic operations and because sample and response arrays remained visible throughout each trial, they could inspect them indefinitely before finalizing their responses (which were unspeeded). In a series of practice trials, all participants correctly performed orthogonal matches for sets of size 3 ,
4, and 5 (with feedback) before advancing to the critical trials.

In critical trials, participants received no feedback about their performance. For high counters, the first critical trial was a sample array of 10 objects. For low-counters, the first critical trial was a sample array with two fewer objects than the participant's highest verbal count. From this starting point, we followed a pre-defined staircasing procedure (i.e. +2 for correct, -1 for incorrect) to determine the size of each sample array until participants (a) produced three incorrect response arrays for sample arrays of the same number (e.g. samples with $\mathrm{N}=15$ objects), (b) correctly matched three arrays numbering 20 or more, or (c) completed 20 critical trials.

\section{Psychophysical model of numerical abilities}

To evaluate the limits of participants' exact numerical representations, we analyzed their distribution of responses using a generative Bayesian data analysis (Gelman, Carlin, Stern, \& Rubin, 2014). This model formalized a process in which participants use an "exact" system (with constant error) for smaller sets and an approximate system (with scalar variability) for larger sets. The number at which participants switched from exact to approximate representations is the participant's switchpoint, our dependent measure.

Formally, for the exact system (i.e., numbers below the switchpoint) we assumed that participants responded from a Cauchy $\left(\mu_{\text {low }}+n, \sigma_{\text {low }}\right)$ distribution, where $n$ is the number of objects in the sample set and $\mu_{\text {low }}$ and $\sigma_{\text {low }}$ are location and scale parameters (so that $\mu_{l o w} \approx 0$ means responses are centered on the true value $n$, and $\sigma_{l o w} \approx 0$ means that responses cluster tightly around the mode $\left.\mu_{\text {low }}+n\right)$. A Cauchy distribution was used because errors in the exact system likely reflect inattention or confusion, and estimation of this distribution is robust to outliers. For the approximate system, we assumed a standard model of approximate number psychophysics (Dehaene, 2011) where subjects respond according to the distribution $\operatorname{Normal}\left(n, w_{i} \cdot n\right)$, where $w_{i}$ is a Weber ratio parameter that varies by individual. Putting these together, the model assumes that, when shown a sample of $n$ objects, participant responses $r$ follow

$$
P\left(r \mid n, w_{i}, \mu_{\text {low }}, \sigma_{\text {low }}\right) \sim \begin{cases}\operatorname{Cauchy}\left(\mu_{\text {low }}+n, \sigma_{\text {low }}\right) & \text { if } n \leq s_{i} \\ \operatorname{Normal}\left(n, w_{i} \cdot n\right) & \text { if } n>s_{i}\end{cases}
$$

where $s_{i}$ is the switchpoint of the $i$ 'th participant. In addition, we included a hierarchical model for participant Weber ratios $w_{i}$, such that $w_{i} \sim \operatorname{Normal}\left(\mu_{W}, \sigma_{W}\right)$ constrained to be positive, which means that we partially pool participant estimates of Weber fraction. We put a uniform

\footnotetext{
${ }^{2}$ Our model requires s to have an upper bound, which we set to the largest number of objects tested (i.e. 40 objects in our test of high-counters' verbal count range). This provides a conservative estimate of high counters' switchpoints, which may be much higher (or unbounded), and allows us to test the sensitivity of these estimates to differences in numerical matching performance.
} 
prior on $s_{i}$ between 1 and $40^{2}$, a standard normal prior on $\mu_{\text {low }}$, and Exponential (1) priors on $\sigma_{\text {low }}, \mu_{W}$, and $\sigma_{W}$ (see Supplementary Materials for details).

This model allowed us to infer the likely distribution of switchpoint values $s_{i}$ from participants' pattern of behavioral responses, while accounting for the uncertainty inherent both to exact enumeration (i.e. a noise parameter for low numbers, shared across participants) and to numerical approximation (i.e. a Weber ratio fit to each participant).

\section{Results}

Whereas high-counters counted to 40 without error on both trials, low-counters' highest verbal counts ranged from 6 to 20 (mean $=12.6$ ), and often differed across the two trials (mean absolute difference $=2.0$ ).

\section{Parallel matching}

In the parallel matching task, high-counters performed at ceiling, correctly matching each of the sample sets (i.e., $\mathrm{N}$ $=3,4,5,10,15)$ on their first attempt. Low-counters were $85 \%$ accurate on their first attempts, with $70 \%$ accuracy on sets larger than five (i.e. $\mathrm{N}=10$ and 15). With one exception, their incorrect responses were within 2 of the correct number (see Figure 1, left), and no participant made more than two errors. When they did make an error, they then showed $100 \%$ accuracy on their second attempt, fully reconstructing the response set without feedback about the magnitude or direction of their error.

\section{Orthogonal matching}

Participants were less accurate in the orthogonal matching task (mean $=51 \%$ correct) than in the parallel matching task (mean $=93 \%$ correct), even for the same cardinalities ( $56 \%$ correct for $\mathrm{N}=3,4,5,10$, or 15; see Figure 1, top).

The model estimated a mean Weber ratio of 0.13 , consistent with Weber ratios found in studies of numerical estimation in adults (Piazza, Izard, Pinel, Le Bihan, \& Dehaene, 2004; Pica et al., 2004), including Tsimane' adults (Gibson, Jara-Ettinger, Levy, \& Piantadosi, 2017). The noise for low numbers was estimated to have a mean of $\mu_{\text {low }}=-0.14$ and a standard deviation of $\sigma_{\text {low }}=0.14$.

The critical question is how participants' switchpoints were related to their verbal counting abilities. Figure 2 (left panel) shows estimated switchpoints as a function of participants' highest verbal counts, and Figure 3 shows the data for each individual low counter. (Because high counters' switchpoints data are less informative on their own (due to potential ceiling effects), their individual plots can be seen in Supplementary Materials.). Although analysis of the response data was blind to participants' counting

abilities, it inferred markedly different switchpoints for low and high counters, solely on the basis of their numerical matching responses. Whereas switchpoints among the low counters averaged below 7 and never exceeded 11 , the average switchpoint among the high counters was over $28(t(17.47)=11.01, p<.0001){ }^{3}{ }^{3}$ Highest verbal count reliably predicted switchpoint, above and beyond any effect of formal education: higher counters had higher switchpoints ( $\beta=.55$, SEM $=0.01, t=5.48, p<.0001$ ). This relationship also held on zero education individuals: highest verbal count reliably predicted switchpoint even when analyzing only those participants with no formal education (i.e. 12 low-counters and 2 high-counters; $\beta=0.40, S E M=0.15, t=2.67, p=.02$; all tests are two-sided).

Importantly, participants' counting abilities and matching abilities were related beyond simple correlation: Low counters' switchpoints fell at or below their highest verbal count (i.e. below the diagonal dotted line) with only one exception, as shown in Figure 2 (left panel). According to Pearson chi-squared tests, this ratio (i.e. above:below) differed significantly from chance $\left(\chi^{2}(1)=9.60, p=.002\right)$. Note that in principle, data points could fall below the line simply due to a floor effect, in which participants' show low switchpoints independent of their counting abilities. However, this possibility is unlikely to explain our results, for two reasons. First, our switchpoint estimates varied widely on the basis of participants' numerical matching performance alone, demonstrating the sensitivity of our tests. Second, in a further test of this possibility, we conducted a permutation test in which we randomized the pairings of participants' highest verbal counts and switchpoints. This procedure respects the marginal distribution of each variable, and therefore allowed us to evaluate what proportion of data points we should expect to fall below the line by chance (i.e., if verbal counting and numerical matching performance were statistically independent). In 10,000 permuted samples, the number of participants whose switchpoints exceeded their highest counts was 7.72 on average and was never as small or smaller than the number we observed (i.e. 1), indicating that the observed pattern is extremely unlikely to occur by chance $(p<.001)$.

Figure 2 (right panel) shows the probability that low counters' switchpoints exceeded their highest verbal counts, calculated using each participants' distribution of switchpoint estimates. Except for one, these switchpoints were below the $50 \%$ threshold (Mean $=11.96 \%$ ), indicating that they were likely within participants' verbal count range. For numbers beyond their highest verbal count, low counters' responses were on average seven times more

\footnotetext{
${ }^{3}$ High counters' switchpoints were estimated based on the range of tested values. Their true switchpoints (and verbal count ranges) are likely much higher, and may be unbounded. Therefore, the difference in switchpoints we observe between groups is likely an underestimate of the true difference.
} 

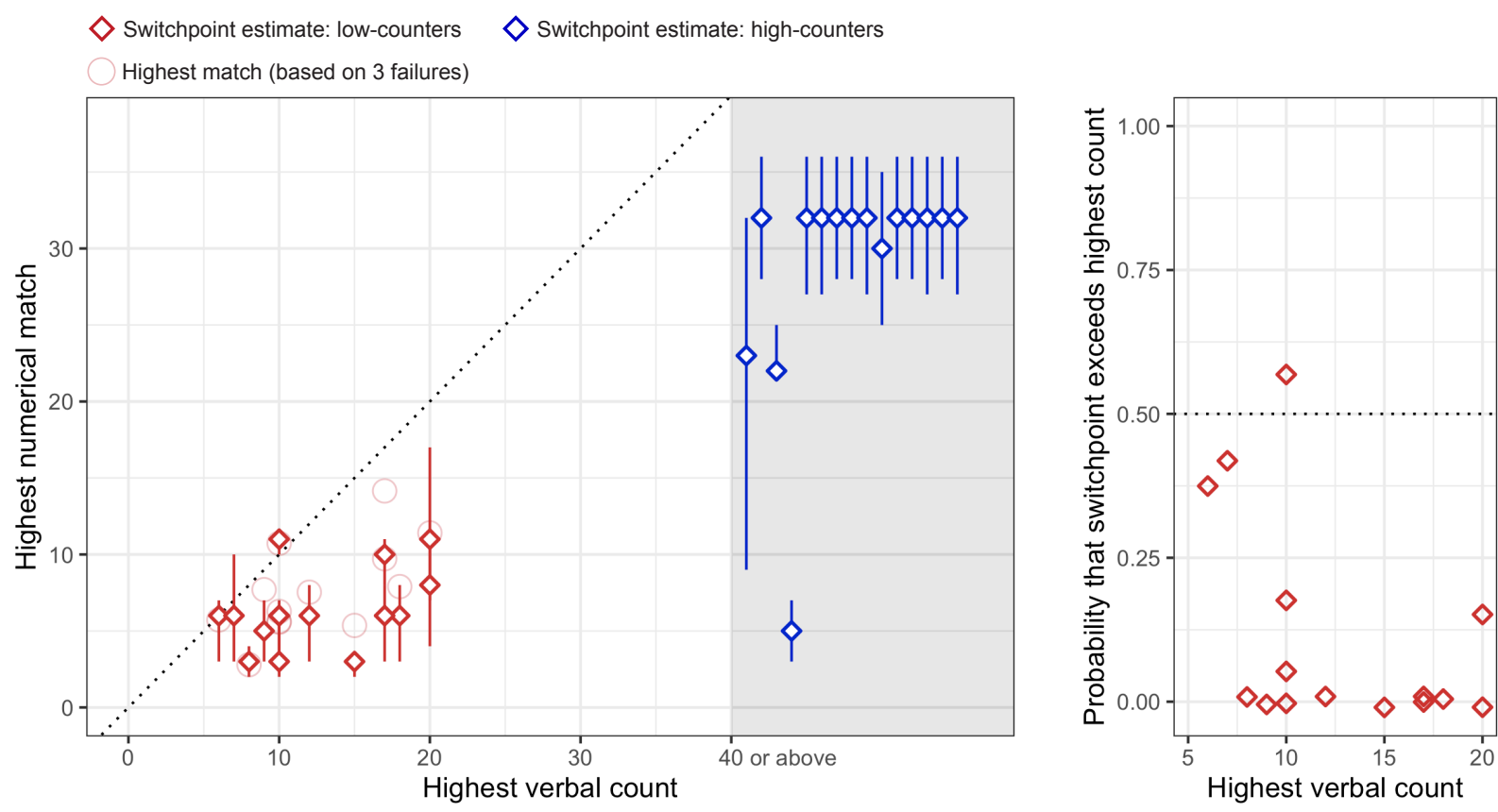

Figure 2: Overview of results. Left: Participants' switchpoints as a function of their highest verbal counts. Diamonds show median estimate and error bars show 50\% confidence intervals. With one exception, all low-counters (red) and high counters (blue) had switchpoints below their highest verbal counts. (See footnote 3 for a discussion of high counters' switchpoints.) The same pattern obtains for an alternative measure of participants' highest numerical match (red circles), based on the set size at which they failed three times. Right: The probability that low-counters' switchpoints exceeded their highest verbal counts.

likely to reflect an approximate system than an exact system.

In addition to our generative model, we also used a simple behavioral criterion to evaluate participants' highest match: the number at which they failed to produce an exact match three times (which also served as one of our stopping criteria during testing). Given the staircase procedure we used for testing, failing three times on sets of $\mathrm{N}$ required a combination of failing on sets of $\mathrm{N}+1$ and succeeding on sets of $\mathrm{N}-2$. We therefore defined highest match as two less than the number at which participants' failed three times. This alternative measure was highly correlated with participants' switchpoints as estimated by the model $\left(R^{2}=0.67, t(11)=2.97, p=.01\right)$. Although these two measures were often identical (see Figure 3), highest match was on average higher than estimated switchpoints (mean difference $=1.77$ ), and therefore provides a more conservative estimate of participants' numerical reproduction abilities. Nevertheless, this alternative measure showed the same relationship to highest count as the switchpoint estimates from our model: With one exception, participants' highest matches were at or below their highest verbal counts (see red circles in Figure 2, left panel, and dashed red lines in Figure 3), and this ratio differed significantly from chance $\left(\chi^{2}=7.69, p=.006\right)$. Low-counters' verbal count range reliably predicted their highest match $(t(11)=2.44, p=.03)$. This alternative measure also revealed the same difference between groups; whereas the highest match for low-counters (by this criteria) was below ten on average (and was always below 15), no high counter failed three times on any number we tested; rather, they all succeeded to make exact numerical matches into the twenties. ${ }^{4}$

\section{Discussion}

In a group of Tsimane' adults, the ability to represent exact numbers was limited to the part of the verbal count list they had mastered. Using a generative model of their responses, we found that participants with a limited verbal count range could reliably match the numerosity of sets only within this verbal range; for larger numbers of objects, they overwhelmingly failed to make exact matches (by two measures), relying instead on numerical approximation. Numerical matching abilities also improved with verbal counting abilities: Participants who showed no upper bound on their verbal count range also showed no upper bound on their numerical matching abilities.

These findings clarify the role of language in number concepts in three ways. First, unlike other isolated groups, our Tsimane' participants succeeded in representing some

\footnotetext{
${ }^{4}$ Two of the fifteen low-counters did not fail three times on the same number within 20 critical trials, and so their data do not appear in Figures 2 or 3
} 


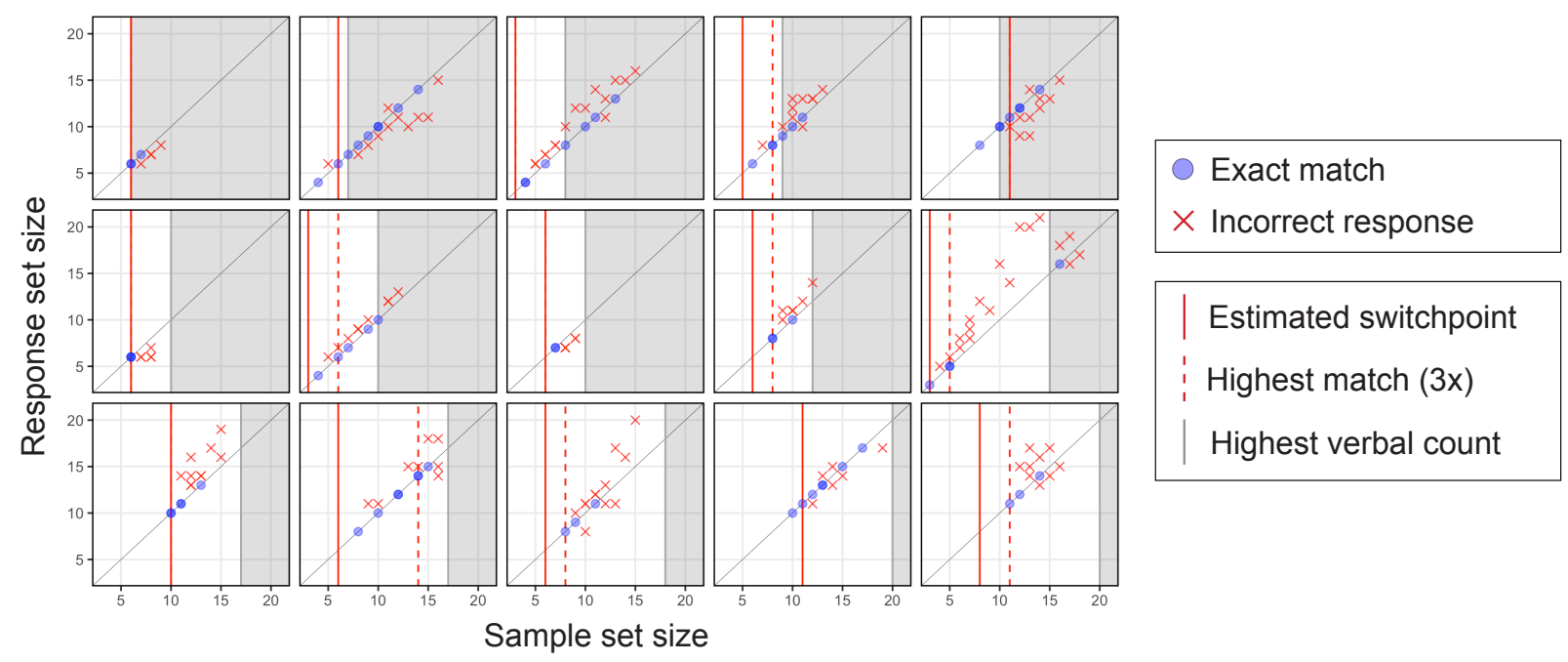

Figure 3: Each plot shows the data of an individual low counter. Blue dots are correct numerical matches and red Xs are incorrect responses. Shaded regions are beyond the participant's verbal count range. With one exception, participants' switchpoints as estimated by the model (solid red lines) were within their verbal count range (unshaded region), as were their highest matches as determined by our $3 \mathrm{x}$ failure criterion (dashed red lines).

cardinalities above four. This success shows that even low counters were not "indifferent to exact numerical equality" (Gelman \& Gallistel, 2004) but rather were attuned to it in both in the orthogonal and parallel matching tasks (in which they succeeded for sets as large as 15). Yet, despite this sensitivity to exact number (and the availability of alternative matching strategies), participants were unable to represent cardinalities beyond their verbal range, even for sets smaller than ten.

Second, the differences in conceptual abilities that we observe cannot reflect broad differences across groups, as our participants shared a common language and culture. In principle, the correlation between participants' numeric abilities could reflect differences in their formal education. However, highest count reliably predicted highest numerical match when we controlled for differences in education, and when we analyzed only the participants with no formal schooling at all. Therefore, this relationship cannot easily be attributed to differences in language, culture, or formal education.

Finally, whereas previous studies have shown (crosscultural) correlations between verbal counting abilities and numerical reproduction abilities, our inferences do not rely on correlation. Rather than simply asking whether one ability predicts the other ability, we also ask whether one ability systematically exceeds the other, allowing us to assess the causal relationship between them. In principle, people could represent "an unbounded set of discrete values...as needed" (Leslie, Gallistel, \& Gelman, 2007) once they are equipped with logic of large exact numbers, whether by experience or by a "preverbal counting mechanism" (Gallistel \& Gelman, 1992). If so, then low counters' numerical matching ranges should have systematically exceeded their verbal counting ranges. We found the opposite pattern, providing the strongest evidence to date that number words play a functional role in representing large exact numbers (Carey, 2009; Le Corre \& Carey, 2007; Carey, 2004; Spelke, 2003; Carey \& Barner, 2019; Piantadosi et al., 2012) and that this role is not all-or-nothing. Rather, the ability to represent large exact numbers depends critically on the availability of the corresponding (verbal) symbols. In this way, the verbal count list may serve not only in the induction of numerical principles, but also in their use. ${ }^{5}$

In interpreting the findings in the Pirahã, Mundurukú, and other isolated groups, some researchers have characterized the verbal count list as a "cultural tool" (Dehaene, 2011) or a "cognitive technology" (Frank et al., 2008; also see Frank \& Barner, 2012). Although these metaphors may be compelling, they do little to clarify whether a verbal count list is necessary for representing large exact numbers. Just as a bicycle is useful but not necessary for transportation, some scholars have argued that "using words to name exact numerosities is useful but not necessary"(Butterworth et al., 2008) for representing large exact numbers, providing an efficient way to encode numerical information that "complements, rather than altering or replacing, nonverbal representations" (Gleitman \& Papafragou, 2012). If such nonverbal representations of large exact numbers exist (e.g. Butterworth et al., 2008; Leslie et al., 2008), they had no effect on the numerical

\footnotetext{
${ }^{5}$ The availability of a verbal count list may be necessary but not sufficient for representing large exact numbers, as evidenced by the large gap between some participants' highest count and highest match.
} 
Pitt, B., Gibson, E., \& Piantadosi S.T. (in press) Psychological Science

abilities of our participants (or of the Pirahã, Mundurukú, or Nicaraguan Homesigners), none of whom showed any sign of "alternative representational strategies" (Gleitman \& Papafragou, 2012). Rather, if the verbal count list is a cognitive technology, it is one that not only facilitates large exact number representations, but enables them, allowing people to maintain precise numerical information over time and space (Frank et al., 2008).

Beyond theories of numerical cognition, these findings also bear on a broader debate about the role of language in cognition (Hume, 1748/2000; Whorf, 2012; Sapir, 1929; Fodor, 1975; Lupyan, 2016; Levinson \& Bowerman, 2001). Linguistic relativity effects have been reported in a variety of domains, including color (Forder \& Lupyan, 2019; Regier \& Kay, 2009), time (Gijssels \& Casasanto, 2017), musical pitch (Dolscheid, Shayan, Majid, \& Casasanto, 2013), and spatial reasoning (Majid, Bowerman, Kita, Haun, \& Levinson, 2004). However, the idea that language shapes thought remains controversial (McWhorter, 2014; Pinker, 1994; Gleitman \& Papafragou, 2012), in part because there are many versions of the "Whorfian hypothesis" (Kay \& Kempton, 1984; Casasanto, 2016). On a strong version, language cannot only change conceptual representations but can also enable new ones (Casasanto, 2016; Gelman \& Butterworth, 2005). The present results reveal such an effect in the domain of number, where language appears to enable representations of exact cardinalities larger than four. To be clear, language may not be the only external symbol system that can enable large exact number concepts (and not all known number words are precisely represented; Landy, Silbert, \& Goldin, 2013). For example, finger counting (Bender \& Beller, 2012), body-part counting (Saxe, 1981), and abacus use (Frank \& Barner, 2012) may also support the development and elaboration of such concepts (Overmann, 2018; Wiese, 2003). Whatever set of symbols people use, their ability to represent large exact numbers extends only as far as their mastery of those symbols.

\section{Acknowledgments}

This work was supported by Grant 1760874 from the National Science Foundation, Division of Research on Learning (to S.P.) and a network grant supporting B.P. from the James S. McDonnell foundation. Special thanks to Tomás Huanca, Esther Conde, and Tania Cruz for coordinating our fieldwork, to our interpreters, Manuel Roca, Robin Nate, and Elías Hiza, for their hard work, and to Kensy Cooperrider for comments on the manuscript.

\section{Open Practices Statement}

This study was not formally pre-registered. De-identified data and analysis scripts are available in the Open Science Framework repository: osf.io/me7w4/

\section{References}

Bender, A., \& Beller, S. (2012). Nature and culture of finger counting: Diversity and representational effects of an embodied cognitive tool. Cognition, 124(2), 156182.

Betancourt, M., \& Girolami, M. (2015). Hamiltonian monte carlo for hierarchical models. Current trends in Bayesian methodology with applications, 79(30), 2-4.

Bloom, P. (1994). Generativity within language and other cognitive domains.

Brannon, E. M. (2005). What animals know about numbers. Handbook of mathematical cognition, 85-107.

Butterworth, B., Reeve, R., Reynolds, F., \& Lloyd, D. (2008). Numerical thought with and without words: Evidence from indigenous australian children. Proceedings of the National Academy of Sciences, 105(35), 1317913184.

Carey, S. (2004). Bootstrapping \& the origin of concepts. Daedalus, 133(1), 59-68.

Carey, S. (2009). The origin of concepts. Oxford University Press.

Carey, S., \& Barner, D. (2019). Ontogenetic origins of human integer representations. Trends in Cognitive Sciences, 23(10), 823-835.

Carpenter, B., Gelman, A., Hoffman, M., Lee, D., Goodrich, B., Betancourt, M., ... Riddell, A. (2016). Stan: A probabilistic programming language. Journal of Statistical Software, 20, 1-37.

Casasanto, D. (2005). Crying" whorf". Science, 307(5716), $1721-1722$.

Casasanto, D. (2016). Linguistic relativity. The Routledge handbook of semantics, 158-174.

Cheyette, S. J., \& Piantadosi, S. T. (2020). A unified account of numerosity perception. Nature Human Behaviour, 4(12), 1265-1272.

Chomsky, N., Keyser, S. J., et al. (1988). Language and problems of knowledge: The managua lectures (Vol. 16). MIT press.

Cooperrider, K., \& Gentner, D. (2019). The career of measurement. Cognition, 191, 103942.

Davidson, K., Eng, K., \& Barner, D. (2012). Does learning to count involve a semantic induction? Cognition, 123(1), 162-173.

Dehaene, S. (1997). The number sense: How the mind creates mathematics. Oxford University Press, USA.

Dehaene, S. (2011). The number sense: How the mind creates mathematics. OUP USA.

Dehaene, S., Dehaene-Lambertz, G., \& Cohen, L. (1998). Abstract representations of numbers in the animal and human brain. Trends in neurosciences, 21(8), 355-361.

Diekmann, Y., Smith, D., Gerbault, P., Dyble, M., Page, A. E., Chaudhary, N., ... Thomas, M. G. (2017). Accurate age estimation in small-scale societies. Proceedings of the National Academy of Sciences, 114(31), 82058210.

Dolscheid, S., Shayan, S., Majid, A., \& Casasanto, D. (2013). The thickness of musical pitch: Psychophysical 
Pitt, B., Gibson, E., \& Piantadosi S.T. (in press) Psychological Science

evidence for linguistic relativity. Psychological science, 24(5), 613-621.

Everett, D. L. (2009). Don't sleep, there are snakes: Life and language in the amazonian jungle. Random House LLC.

Feigenson, L., Dehaene, S., \& Spelke, E. (2004). Core systems of number. Trends in cognitive sciences, $8(7)$, 307-314.

Fodor, J. (1975). The language of thought. Cambridge, MA: Harvard University Press.

Forder, L., \& Lupyan, G. (2019). Hearing words changes color perception: Facilitation of color discrimination by verbal and visual cues. Journal of Experimental Psychology: General, 148(7), 1105.

Frank, M. C., \& Barner, D. (2012). Representing exact number visually using mental abacus. Journal of Experimental Psychology-General, 141(1), 134.

Frank, M. C., Everett, D. L., Fedorenko, E., \& Gibson, E. (2008). Number as a cognitive technology: Evidence from pirahã language and cognition. Cognition, 108(3), 819-824.

Frank, M. C., Fedorenko, E., Lai, P., Saxe, R., \& Gibson, E. (2012). Verbal interference suppresses exact numerical representation. Cognitive psychology, 64(1-2), 74-92.

Gallistel, C. R., \& Gelman, R. (1992). Preverbal and verbal counting and computation. Cognition, 44(1-2), 43-74.

Gelman, A., Carlin, J. B., Stern, H. S., \& Rubin, D. B. (2014). Bayesian data analysis (Vol. 2). Taylor \& Francis.

Gelman, R., \& Butterworth, B. (2005). Number and language: how are they related? Trends in cognitive sciences, 9(1), 6-10.

Gelman, R., \& Gallistel, C. R. (2004). Language and the origin of numerical concepts. Science, 306(5695), 441-443.

Gibson, E., Jara-Ettinger, J., Levy, R., \& Piantadosi, S. (2017). The use of a computer display exaggerates the connection between education and approximate number ability in remote populations. Open Mind, 2(1), 37-46.

Gijssels, T., \& Casasanto, D. (2017). Conceptualizing time in terms of space: Experimental evidence. Cambridge handbook of cognitive linguistics, 651-668.

Gleitman, L., \& Papafragou, A. (2012). New perspectives on language and thought. The Oxford handbook of thinking and reasoning, 2, 543-568.

Gordon, P. (2004). Numerical cognition without words: Evidence from Amazonia. Science, 306(5695), 496.

Henrich, J., Heine, S. J., \& Norenzayan, A. (2010). The weirdest people in the world? Behavioral and Brain Sciences, 33(2-3), 61-83.

Hoffman, M. D., \& Gelman, A. (2014). The no-u-turn sampler: adaptively setting path lengths in hamiltonian monte carlo. Journal of Machine Learning Research, 15(1), 1593-1623.

Huanca, T. (2008). Tsimane oral tradition, landscape, and identity in tropical forest. SEPHIS, South-South Exchange Programme for Research on the History of Development.
Hume, D. (1748/2000). An enquiry concerning human understanding. New Jersey: Prentice Hall.

Hume, D. (1978). A treatise of human nature [1739]. British Moralists, 1650, 1800.

Jara-Ettinger, J., Piantadosi, S., Spelke, E. S., Levy, R., \& Gibson, E. (2017). Mastery of the logic of natural numbers is not the result of mastery of counting: Evidence from late counters. Developmental science, 20(6), e12459.

Kay, P., \& Kempton, W. (1984). What is the sapir-whorf hypothesis? American anthropologist, 86(1), 65-79.

Landy, D., Silbert, N., \& Goldin, A. (2013). Estimating large numbers. Cognitive science, 37(5), 775-799.

Laurence, S., \& Margolis, E. (2007). Linguistic determinism and the innate basis of number. The Innate Mind, 3, 139-169.

Le Corre, M., \& Carey, S. (2007). One, two, three, four, nothing more: An investigation of the conceptual sources of the verbal counting principles. Cognition, 105(2), 395-438.

Leslie, A. M., Gallistel, C., \& Gelman, R. (2007). Where integers come from. The innate mind: Foundations and the future, 3, 109-149.

Leslie, A. M., Gelman, R., \& Gallistel, C. (2008). The generative basis of natural number concepts. Trends in cognitive sciences, 12(6), 213-218.

Levinson, S. C., \& Bowerman, M. (2001). Language acquisition and conceptual development (No. 3). Cambridge University Press.

Lupyan, G. (2016). The centrality of language in human cognition. Language Learning, 66(3), 516-553.

Majid, A., Bowerman, M., Kita, S., Haun, D. B., \& Levinson, S. C. (2004). Can language restructure cognition? the case for space. Trends in cognitive sciences, 8(3), 108-114.

McWhorter, J. H. (2014). The language hoax: Why the world looks the same in any language. Oxford University Press, USA.

Overmann, K. A. (2018). Constructing a concept of number.

Pahl, M., Si, A., \& Zhang, S. (2013). Numerical cognition in bees and other insects. Frontiers in psychology, 4, 162.

Piantadosi, S. T., Tenenbaum, J. B., \& Goodman, N. D. (2012). Bootstrapping in a language of thought: A formal model of numerical concept learning. Cognition, 123(2), 199-217.

Piazza, M., Izard, V., Pinel, P., Le Bihan, D., \& Dehaene, S. (2004). Tuning curves for approximate numerosity in the human intraparietal sulcus. Neuron, 44(3), 547-555.

Pica, P., Lemer, C., Izard, V., \& Dehaene, S. (2004). Exact and approximate arithmetic in an amazonian indigene group. Science, 306(5695), 499-503.

Pinker, S. (1994). The language instinct, william morrow and company. Inc., New York.

Regier, T., \& Kay, P. (2009). Language, thought, and color: Whorf was half right. Trends in cognitive sciences, 13(10), 439-446. 
Sapir, E. (1929). The status of linguistics as a science. Language, 207-214.

Sarnecka, B. W., Goldman, M. C., \& Slusser, E. B. (2015). How counting leads to children's first representations of exact, large numbers. Oxford handbook of numerical cognition, 291-309.

Saxe, G. B. (1981). Body parts as numerals: A developmental analysis of numeration among the oksapmin in papua new guinea. Child development, 306-316.

Schneider, R. M., \& Barner, D. (2020). Children use one-to-one correspondence to establish equality after learning to count. 42nd Annual Meeting of the Cognitive Science Society.

Spaepen, E., Coppola, M., Spelke, E. S., Carey, S. E., \& Goldin-Meadow, S. (2011). Number without a lan- guage model. Proceedings of the National Academy of Sciences, 108(8), 3163-3168.

Spelke, E. S. (2003). What makes us smart? core knowledge and natural language. Language in mind: Advances in the study of language and thought, 277-311.

Team, S. D., et al. (2016). Rstan: the $\mathrm{r}$ interface to stan. $R$ package version, 2(1).

Whorf, B. L. (2012). Language, thought, and reality: Selected writings of benjamin lee whorf. Mit Press.

Wiese, H. (2003). Iconic and non-iconic stages in number development: the role of language. Trends in cognitive sciences, 7(9), 385-390.

Wynn, K. (1992). Children's acquisition of the number words and the counting system. Cognitive Psychology, 24(2), 220-251. 


\title{
Supplementary Material
}

\section{Exact number concepts are limited to the verbal count range}

\author{
Benjamin Pitt, Edward Gibson, \& Steven Piantadosi
}

\section{Parallel matching task}

To begin the parallel matching task, an experimenter seated across from the participant laid out two white buttons (arranged left-right) and explained that the participant was to make a set of pebbles with the exact same number of objects. The experimenter then demonstrated the correct response by moving two pebbles from the participant's pile into alignment with the two buttons, making two parallel rows of two objects. Then in a series of five trials, the experimenter increased the sample array from 2 buttons to $3,4,5,10$, and then 15 buttons. Participants were given unlimited time to complete each match, and trials ended only after the participant verbally indicated that they had finished, at which point the response array was removed. When the response was correct, participants received verbal confirmation that their response was accurate. When participants produced a response array that differed in number from the sample, the discrepancy was pointed out and the trial was repeated. All participants completed the five trials of the parallel matching task correctly before advancing to the orthogonal matching task.

\section{Orthogonal matching task}

Like the parallel matching task, the orthogonal matching task began with a demonstration using a set size of two. The experimenter placed two buttons on the table (arranged front-back) and explained that the participant was so do the same as before: make a lateral array of pebbles with the same number of objects as the sample. In warm-up trials, participants made orthogonal matches to sets of 3 , 4 , and 5 buttons. If participants produced a response array in these trials that differed in number from the sample, the discrepancy was pointed out and the trial was repeated. All participants completed the three warm-up trials correctly before advancing to the critical trials. For the critical trials, low-counters began with an array of two less than their highest count and high-counters began with an array of ten. From this starting point, all participants followed the same staircasing procedure: after a correct response, the set size was increased by two; after an incorrect response, the set size was decreased by one (i.e. $+2,-1)$.
To ensure that participants evaluated the cardinality of each sample array independently of the preceding arrays, at the end of each trial we (i) removed the response array (and reincorporated it into the larger pile of pebbles) and (ii) removed an arbitrary subset of buttons from the sample array before making the subsequent sample array. This aspect of the procedure allowed the experimenter to change the cardinality of the sample set (i.e. add two or subtract one button) out of sight of participants, making it difficult for participants to track the changes to the sample array or to infer the accuracy of their responses from those changes.

Participants were instructed to take as much time as required to ensure that their array of objects had exactly the same number of objects as in the sample array, and were free to touch the objects as needed. Each trial ended only when the participant verbally indicated that they had finished, and no feedback was given during the critical trials.

\section{Modeling}

Posterior distributions were inferred using a No-U-Turn sampler in Stan (Hoffman \& Gelman, 2014; Carpenter et al., 2016; Team et al., 2016) with four chains of 10000 samples. In order to create a model with only continuous parameters, we marginalized out each participant's cutoff parameter $s_{i}$, and then computed posterior samples of those $s_{i}$ from samples of other parameters. Because our behavioral responses were discrete, we computed the probability of a response $r$ under either the Cauchy or Normal distribution as the total probability mass between $r-\frac{1}{2}$ and $r+\frac{1}{2}$. Our implementation used a non-centered parameterization of subject effects (Betancourt \& Girolami, 2015) and was run with adapt_delta $=0.9999$. With these parameters, the model encountered 209 divergent transitions in 10000 samples, but examination of a pairs plot did not reveal any regions of obvious difficulty or bias in the model. Overall, convergence was assessed by examination of the traces and computation of $\hat{R}$, which was approximately 1 . The code for this model is available at osf.io/me $7 \mathrm{w} 4 /$. 
Pitt, B., Gibson, E., \& Piantadosi S.T. (in press) Psychological Science

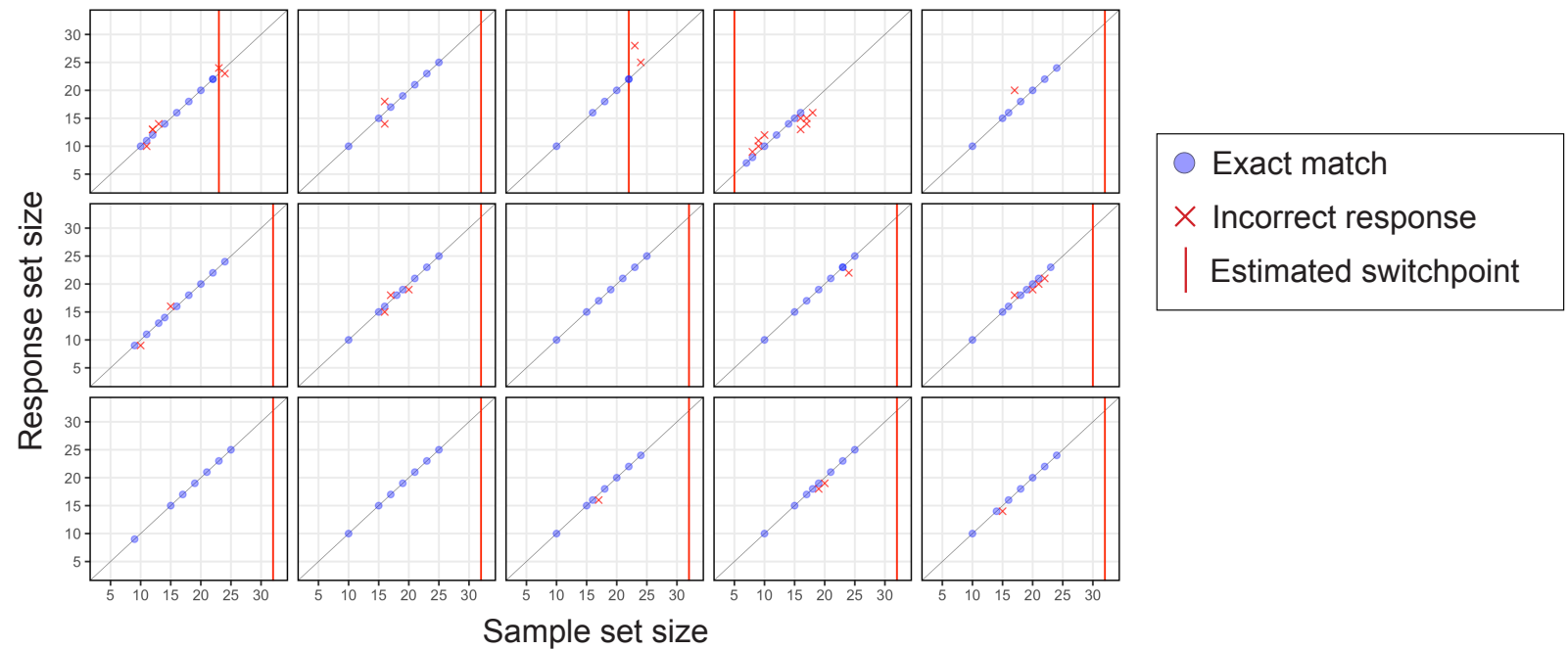

Figure S1. Each plot shows the data of an individual high counter, with unknown highest verbal count (at least 40). Blue dots are correct numerical matches and red Xs are incorrect responses. Vertical red lines show participants' switchpoints as estimated by the model. 